\section{Review Article}

Journal of Epilepsy Research pISSN 2233-6249 / elSSN 2233-6257

Received January 27, 2017

Accepted May 17, 2017

Corresponding author: Soonhak Kwon Department of Pediatric Neurology, Kyungpook National University Children's Hospital, and Kyungpook National University Schoo of Medicine, Hogook-Ro 807, Book-Gu, Daegu, 41404, Korea

Tel. $+82-53-200-2168$

Fax. +82-53-200-2029

E-mail; shkwon@knu.ac.kr

\title{
The Clinical Spectrum of Benign Epilepsy with Centro-Temporal Spikes: a Challenge in Categorization and Predictability
}

\author{
Yun Jeong Lee, Su Kyeong Hwang, Soonhak Kwon \\ Department of Pediatric Neurology, Kyungpook National University Children's Hospital, Kyungpook National University \\ School of Medicine, Daegu, Korea
}

\begin{abstract}
Benign epilepsy with centro-temporal spikes (BECTS) is the most common type of focal epilepsy in children; it is age-dependent and presumably genetic. Traditionally, children with BECTS have a very good prognosis, even without medical treatment, and are thought to show no neurological symptoms or cognitive deficits. However, many previous studies have shown that BECTS can present with various clinical and electroencephalographic characteristics that are commonly associated with neuropsychological deficits, including linguistic, cognitive, and behavioral impairment. The degree of the neuropsychological deficits appears to depend on the sleep cycle and the localization of epileptiform discharges. Furthermore, based on neurobiological studies, a complex interplay between the processes of brain maturation and the involvement of genes that confer susceptibility may contribute to a variety of different childhood epileptic syndromes with various neuropsychological deficits. Thus, BECTS, atypical benign focal epilepsy during childhood, status epilepticus of BECTS, Landau-Kleffner syndrome, and epileptic encephalopathy with continuous spike-and-wave during sleep are all considered different entities, but are part of a single spectrum of disorders. In clinical practice, we have to consider BECTS as benign only when there are no or only mild neuropsychological deficits before medical treatment. (2017;7:1-6)
\end{abstract}

Key words: Benign epilepsy with centro-temporal spikes, Atypical benign focal epilepsy, Landau-Kleffner syndrome, Continuous spike-and-wave during sleep, Electrical status epilepticus during slow-wave sleep

\section{Introduction}

Benign epilepsy with centro-temporal spikes (BECTS) is the most common type of focal epilepsy in children. It is known to be age-dependent, presumably genetic, and mainly occurs at developmentally critical ages. Age of onset ranges from one to 14 years and the prevalence is about $15-20 \%$ in children younger than 15 years of age. ${ }^{1,2}$ Generally, BECTS is characterized by infrequent focal sensorimotor seizures in the face during sleep, which may secondarily generalize, along with spike-wave discharges, reflecting nonlesional cortical excitability from rolandic regions. The prognosis is usually considered to be excellent. Over the past years, however, some investigators have questioned whether BECTS is indeed benign, considering the variety of different presentations associated with the disorder. It is not uncommon for BECTS to be associated with neuropsychological deficits, such as linguistic, cognitive, and behavioral impairment. ${ }^{3-7}$ In partic- ular, reading difficulties and speech/language disorders are more common in children with BECTS than in healthy controls. ${ }^{8}$ Various neuropsychological deficits seem to be very dependent on the sleep-wake cycle and spike index, as well as the predominant localization of epileptiform discharges. ${ }^{9-15}$ Furthermore, the frequency of epileptiform discharges is closely related not only to the degree of neuropsychological deficits, but also to an atypical evolution of BECTS. The atypical evolution of BECTS can result in atypical benign childhood focal epilepsy (ABCFE), status epilepticus of BECTS (SEBECTS), Landau-Kleffner syndrome (LKS), and epileptic encephalopathy with continuous spike-and-wave during sleep (CSWS), which are considered as different entities, but are part of a single spectrum of disorders. ${ }^{16}$ Genetic predisposition with complex modes of inheritance has long been advocated for this wide range of epileptic syndromes, even though unraveling the pathogenic mechanisms that underlie these conditions remains a challenge. Considering the vari- 
ous possible developmental trajectories of the disorder, future research needs to work on evidence-based categorization of BECTS and related epileptic syndromes.

\section{Atypical features and atypical evolution of benign childhood epilepsy with centro-temporal spikes}

Typical BECTS, known as BECTS pure, is an age-dependent, presumably genetic, male predominant, focal epilepsy syndrome in children. The cardinal features typically consist of single or infrequent, brief hemifacial sensory motor seizures, usually during sleep. These seizures are often associated with oropharyngolaryngeal symptoms, such as making strange noises, speech arrest, and salivation. In about half of children with BECTS, the seizures evolve into generalized tonic-clonic seizures. The interictal electroencephalogram (EEG) is characterized by high-voltage focal or multifocal spikes, mainly in rolandic regions, showing a tendency to spread to adjacent regions. This neural activity is common during sleep. There are no, or very few, linguistic or neuropsychiatric deficits. Seizures and EEG features usually resolve within a few years of onset, and typically before the age of 15 or 16 years.

Atypical features in BECTS comprise many clinical and electrophysiological findings. The seizures only occur in the daytime, and Todd's paralysis can be prolonged or even present as status epilepticus. The EEG data can show an atypical morphology of epileptiform discharges, an abnormal background, unusual location, and focal or bilateral synchronous discharges, including the $3 \mathrm{~Hz}$ spike and wave complexes. ${ }^{16-21}$ The atypical features seem to be related to the onset of the seizures. ${ }^{22-25}$ In addition, previous studies have shown that many children with atypical features are likely to have linguistic, learning, or behavioral difficulties. ${ }^{15-17,20,25-28}$ The common comorbidities are listed in Table 1.

Table 1. Comorbidities frequently associated with BECTS

\begin{tabular}{l}
\hline Linguistic impairment \\
Lexical-syntactic, semantic/pragmatic, phonological processing, \\
aphasia, inarticulation \\
Cognitive/learning difficulties \\
Executive, attention, memory, visuo-motor, dyslexia, dyscalculia \\
Behavioral difficulties \\
ADHD, aggression, oppositional-defiant, and others \\
Emotional difficulties \\
Anxiety, depression \\
Other neuropsychiatric impairments \\
Autism
\end{tabular}

BECTS, benign epilepsy with centro-temporal spikes; ADHD, attention deficit hyperactivity disorder.
The atypical evolution of the disorder seems to be closely related to the sleep-wake cycle and the frequency of epileptiform discharges. Individuals with an atypical evolution tend to have moderate to severe linguistic, cognitive, or neuropsychological deficits, which can be permanent. The EEG data usually show a marked increase in abundance and a bilateral synchronization of epileptiform discharges in the rolandic area, or otherwise ripples superimposed on rolandic spikes, which may become continuous during NREM sleep. 10,11,16,24,29 Electrical status epilepticus during slow-wave sleep (ESES) is characterized by paroxysmal or strong activation of diffuse or bilaterally synchronous spikes and waves at about 1-3.5 Hz during NREM sleep (Fig. 1). Sometimes, BECTS can evolve into ABFEC, SEBECTS, LKS, and CSWS. The degree of the neuropsychological deficits and the prognosis of each condition are illustrated in Fig. 2.

Over the past years, these conditions have been considered as different disease entities but part of a single spectrum of disorders. Genetic predisposition has long been advocated for this wide range of syndromes. Several studies have attempted to determine the association between atypical rolandic epilepsy and known genes. Although associations remain speculative, putative genes include fork head box protein P2 (FOXP), which has been related to speech and language development, and sushi repeat-containing protein, $X$-linked 2 (SRPXZ), which is involved in conditions of the rolandic or Sylvian areas, such as speech and language disorders, cognitive difficulties, and neuronal migration disorders. A third relevant gene is elongation factor protein 4 (ELP4), which is associated with language impairment, autism spectrum disorder, and mental retardation.

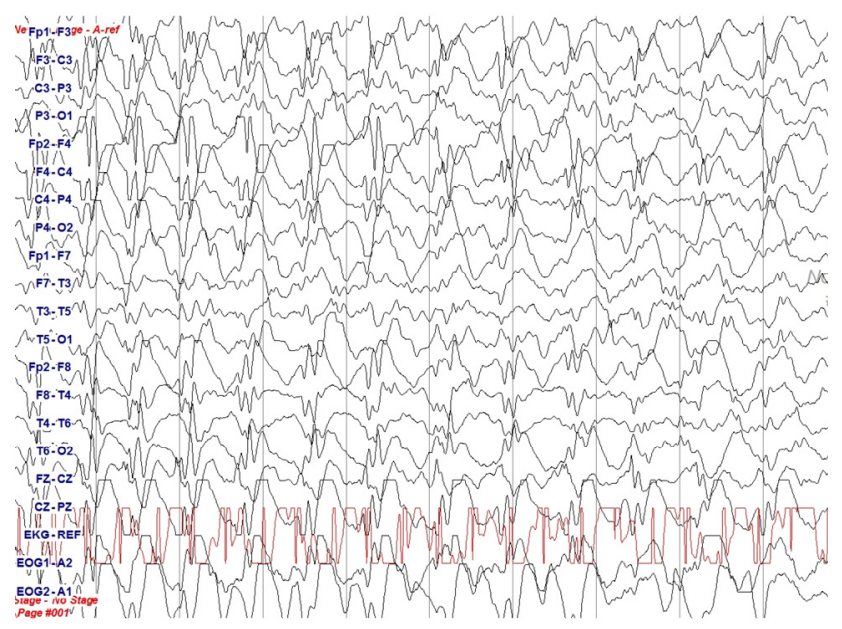

Figure 1. Diffuse or bilaterally synchronous spike and waves at about 1-3.5 $\mathrm{Hz}$ during non-rapid eye movement (NREM) sleep, which are characteristic of electrical status epilepticus during slow-wave sleep (ESES). 


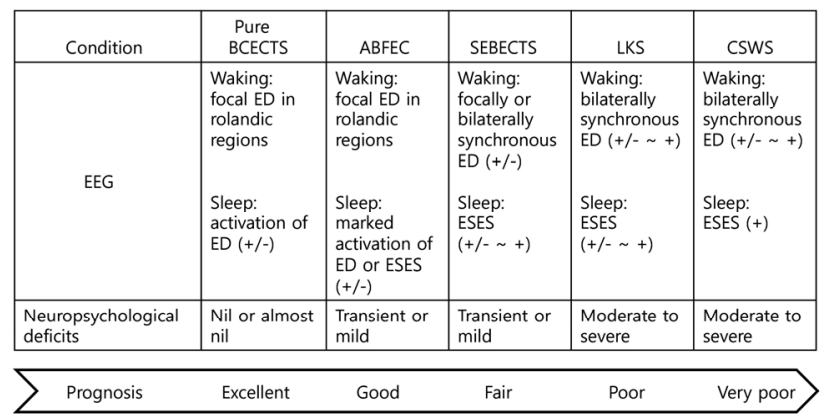

Figure 2. Atypical evolutions of benign epilepsy with centro-temporal spikes (BECTS), their electroencephalogram (EEG), neuropsychological features, and prognosis. ABFEC, atypical benign focal epilepsy of childhood; SEBECTS, status epilepticus of benign epilepsy with centrotemporal spikes; LKS, Landau-Kleffner syndrome; CSW, epileptic encephalopathy with continuous spike-and-wave during sleep; ED, epileptiform discharge; ESES, electrical status epilepticus during slow-wave sleep.

Further relevant genes include the N-methyl-D-aspartate (NMDA) receptor subunit-encoding genes (GRIN2A and GRIN2B), linked to speech and language, cognitive impairment, and behavioral difficulties; the proline-rich transmembrane protein 2 (PRRT2) gene contributing to a wide spectrum of neurological diseases, ranging from mental retardation to paroxysmal neurological conditions such as paroxysmal kinesigenic dyskinesia (PKD), epilepsy and migraine; and the gamma-aminobutyric acid type $A$ receptor subunit genes (GABAA-R and GABRGZ), which have been associated with various neuropsychiatric conditions. ${ }^{30-40}$ Very recent studies showed evidence that children with typical or atypical features share known epilepsy genes, including GRIN2A and PRRT2, which can considered as different clinical phenotypes of a single pathological entity situated at the crossroads of speech and language disorders, cognitive disorders, and epilepsy. ${ }^{41,42}$

\section{Atypical benign focal epilepsy of childhood/ Pseudo-Lennox syndrome}

ABFEC is a form of idiopathic focal epilepsy characterized by normal development prior to the onset of seizures and multiple seizure types. Beside the typical sensorimotor seizures of BECTS, various other types of seizures may also occur, such as secondarily generalized tonic-clonic seizures, atypical absence, myoclonic seizures, atonic seizures, and negative myoclonus at a later stage. Individuals with ABFEC are more likely to have neurocognitive, speech and language, or behavioral deficits. The waking EEG usually resembles that of BECTS, but the sleeping EEG shows the ESES pattern. The electro- clinical state is clearly related to an individual's neuropsychological deficits. The associated seizures tend to be resistant to many antiepileptic agents; however, they usually disappear before adolescence and the neuropsychological deficits recede with the remission of ESES. ${ }^{27,43,44}$ Pseudo-Lennox syndrome is a more severe form of ABFEC with respect to clinical features, seizure outcome, and neuropsychiatric symptoms. Some patients remain mentally retarded even after the remission of the seizures. ${ }^{45-47}$

\section{Status epilepticus of BECTS}

SEBECTS refers to status epilepticus that can be convulsive or non-convulsive, and either generalized or focal. Besides hemifacial contraction, the seizures may present with dysfunctions of the lip, tongue, and pharynx, including speech arrest, dysarthria, excessive drooling, oromotor dyspraxia, and swallowing difficulties. 17,27,46,48 The interictal EEG usually shows focally or bilaterally synchronous sharp waves or sharp and slow wave complexes with predominance in the rolandic area. The epileptiform discharges may become continuous when the individual falls asleep. Ultimate neurocognitive outcome appears good when the disorder is appropriately treated.

\section{Landau-Kleffner syndrome}

LKS, known as acquired epileptic aphasia, is an epileptic encephalopathy characterized by various types of seizures, insidious or sudden onset acquired aphasia with verbal auditory agnosia, and neurocognitive regression that is associated with distinctive EEG abnormalities. Incidence of LKS in children aged 5-14 years is about one in a million and prevalence in children aged $5-19$ years is one in about 300,000-410,000 in Japan. ${ }^{49}$ LKS is considered as one of the rarest but most severe genetic childhood focal epilepsies. Alongside other putative genes, mutations in the GRIN2A gene (encoding the $\mathrm{N}$-methyl-D-aspartate glutamate receptor alpha2 subunit, GluN2A) are thought to be related to this condition. ${ }^{41,50}$ Besides focal motor seizures, children with LKS may have generalized tonic-clonic seizures, atypical absence, atonic, and other type of seizures. The majority of children with LKS present with expressive aphasia, and more than half of these children have problems with receptive language, auditory processing, auditory working memory, and verbal memory, as well as learning difficulties and attentional and behavioral problems. ${ }^{51}$ Benign EEG patterns, like focal epileptiform discharges, may evolve into ESES during sleep in about half of children with $\mathrm{LKS}^{52} \mathrm{~A}$ previous study showed that more than $80 \%$ of children with LKS had bilateral epileptiform discharges in the perisylvian cortex, an 
area of the cerebral cortex associated with speech, language, and auditory processing; and about 20\% demonstrated a unilateral perisylvian hotspot that triggered secondary bilateral synchronization. ${ }^{53}$ The degree of neurocognitive deterioration may correlate with the site and spread of the epileptiform discharges. However, further studies are necessary to elucidate whether these bioelectrical abnormalities play a causal role in children with LKS. Adequate and early medical intervention may avoid language and cognitive impairments. Long-term outcome for LKS is not poor, but seizures and EEG abnormalities do not always disappear. Most children with ESES experience permanent language or cognitive impairment, depending on how well their seizures and EEG patterns respond to treatment. Overall, individuals with LKS report a poor quality of life, mainly due to language or cognitive difficulties. ${ }^{54}$

Acquired opercular epilepsy with oromotor dysfunction is a condition that is difficult to categorize because the clinical features look similar to SEBECTS and LKS. Acquired opercular epilepsy with oromotor dysfunction presents with mixed types of seizures and prolonged episodes of dysarthria, a gradual decrease in verbal output, oromotor dysfunction, and deterioration in cognitive function over several weeks to years. The EEG shows remarkably activated sharp, or sharp-slow discharges, or ESES patterns on rare occasions during NREM sleep. Magnetic resonance imaging of the brain is usually normal.

\section{Epileptic encephalopathy with continuous spike-and-waves during slow-wave sleep}

CSWS or ESES is an age-related, epileptic encephalopathy that presents at the extreme end of atypical BECTS. It is typically characterized by various types of seizures, CSWS or ESES shown on EEG during NREM sleep, and global regression of linguistic, cognitive function, and behavior. The onset of seizures varies, but the seizures tend to peak at about five years of age, before evolving into epileptic encephalopathy with ESES within 1-2 years. ${ }^{55}$ At first, the seizures may be simple focal motor, complex focal, absence, or myoclonic, and usually occur at night. They can last for more than 30 minutes. The EEG usually shows multifocal epileptiform discharges or bilaterally synchronous sharp or spike-wave discharges. Subsequently, the seizures become frequent and predominantly nocturnal. Various types of seizures then emerge. They include hemiconvulsive, generalized tonic-clonic seizures (GTCS), absence seizures, drop attacks, and convulsive or non-convulsive status epilepticus. The EEG starts showing diffuse paroxysms and a continuous pattern of ESES, which eventually occupies at least $85 \%$ of NREM sleep. ${ }^{27}$ Linguistic, neurocognitive decline, and neuropsychiatric features, such as autism, are commonly associated with this condition; the development of these features is largely dependent on the EEG pattern, including the location and abundance of epileptiform discharges. However, the pathophysiological mechanisms are still unclear. It appears that the longer the duration of ESES, the poorer the outcome is. ${ }^{25,56-63}$ Children with CSWS show differences in the degree and type of neurocognitive regression, the features of seizures, and EEG abnormalities. Therefore, it is not appropriate to generalize about this type of epilepsy. Several treatment regimens for atypical BECTS, especially LKS or ESES, have been used to improve cognitive outcome, though these are not evidence-based. Treatment options include benzodiazepines, sulthiame, new antiepileptic drugs such as lamotrigine, topiramate, levetiracetam, a ketogenic diet, steroid or methylprednisolone pulse therapy, intravenous immunoglobulin, and even surgical interventions. s1,64,65 $^{-15}$

In conclusion, BECTS is the most common, presumably genetic, type of focal epilepsy in children. It can develop in various ways, leading to mild or severe neuropsychological deficits, including linguistic, cognitive, and behavioral impairment. The seizures appear to depend on the sleep cycle, and the predominant localization and abundance of epileptiform discharges. A complex interplay between the processes of brain maturation and the effects of specific genes seems to contribute to a variety of different childhood epileptic syndromes. BECTS, atypical BECTS, SEBECTS, LKS, and CSWS can all be considered different entities that are nonetheless part of a single spectrum of disorders. However, much larger cohort studies are needed using comprehensive evaluation including electro-clinical tests and whole or targeted exome sequencing, to obtain a clearer picture of what might be directly causing, or contributing to the diverse phenotypes of this spectrum of disorders.

\section{References}

1. Panayiotopoulos C. A clinical guide to epileptic syndromes and their treatment. London: Springer-Verlag London; 2007. 286 p.

2. Watanabe K. Benign partial epilepsies. In: Wallace SJ, Farrell K, ed. Epilepsy in children. 2nd ed. London: Arnold; 2004. p. 199-220.

3. Weglage J, Demsky A, Pietsch M, Kurlemann G. Neuropsychological, intellectual, and behavioral findings in patients with centrotemporal spikes with and without seizures. Dev Med Child Neurol 1997;39:646-51.

4. Staden U, Isaacs E, Boyd SG, Brandl U, Neville BG. Language dysfunction in children with Rolandic epilepsy. Neuropediatrics 1998;29:242-8. 
5. Ay Y, Gokben S, Serdaroglu G, et al. Neuropsychologic impairment in children with rolandic epilepsy. Pediatr Neurol 2009;41:359-63.

6. Danielsson J, Petermann F. Cognitive deficits in children with benign rolandic epilepsy of childhood or rolandic discharges: a study of children between 4 and 7 years of age with and without seizures compared with healthy controls. Epilepsy Behav 2009;16:646-51.

7. Kavros PM, Clarke T, Strug $\amalg$, Halperin JM, Dorta NJ, Pal DK. Attention impairment in rolandic epilepsy: systematic review. Epilepsia 2008;49:1570-80.

8. Clarke T, Strug $\sqcup$, Murphy PL, et al. High risk of reading disability and speech sound disorder in rolandic epilepsy families: case-control study. Epilepsia 2007;48:2258-65.

9. Deonna $T$, Zesiger $P$, Davidoff $V$, Maeder M, Mayor C, Roulet E. Benign partial epilepsy of childhood: a longitudinal neuropsychological and EEG study of cognitive function. Dev Med Child Neurol 2000;42:595-603.

10. Nicolai J, van der Linden I, Arends JB, et al. EEG characteristics related to educational impairments in children with benign childhood epilepsy with centrotemporal spikes. Epilepsia 2007;48:2093-100.

11. Kanemura H, Sano F, Aoyagi K, Sugita K, Aihara M. Do sequential EEG changes predict atypical clinical features in rolandic epilepsy? Dev Med Child Neurol 2012;54:912-7.

12. Wendorff J, Przygocka J, Juchniewicz B. Cognitive disturbances in rolandic epilepsy--correlation with electoencephalographic patterns. Przegl Lek 2006;63 Suppl 1:14-7.

13. Zhao X, Chi Z, Chi L, Shang W, Liu X. Clinical and EEG characteristics of benign rolandic epilepsy in Chinese patients. Brain Dev 2007;29:13-8.

14. Kwon S, Hwang TG, Lee J, Kim DK, Seo HE. Benign childhood epilepsy with centrotemporal spikes: to treat or not to treat. J Epilepsy Res 2013;3:1-6.

15. Kwon S, Seo HE, Hwang SK. Cognitive and other neuropsychological profiles in children with newly diagnosed benign rolandic epilepsy. Korean J Pediatr 2012;55:383-7.

16. Fejerman N. Atypical rolandic epilepsy. Epilepsia 2009;50 Suppl 7:9-12.

17. Aicardi J. Atypical semiology of rolandic epilepsy in some related syndromes. Epileptic Disord 2000;2 Suppl 1:S5-9.

18. Besenyei M, Varga E, Fekete I, et al. EEG background activity is abnormal in the temporal and inferior parietal cortex in benign rolandic epilepsy of childhood: a LORETA study. Epilepsy Res 2012;98:44-9.

19. Gregory DL, Farrell K, Wong PK. Partial status epilepticus in benign childhood epilepsy with centrotemporal spikes: are independent right and left seizures a risk factor? Epilepsia 2002;43:936-40.

20. Verrotti A, Latini $G$, Trotta $D$, et al. Typical and atypical rolandic epilepsy in childhood: a follow-up study. Pediatric Neurol 2002;26:26-9.

21. Striano P, Vari MS. Todd paralysis in rolandic epilepsy. Pediatr Neurol Briefs 2015;29:50.

22. Callenbach PM, Bouma PA, Geerts AT, et al. Long term outcome of benign childhood epilepsy with centrotemporal spikes: Dutch study of epilepsy in childhood. Seizure 2010;19:501-6.

23. You SJ, Kim DS, Ko TS. Benign childhood epilepsy with centro-temporal spikes (BCECTS): early onset of seizures is associated with poorer response to initial treatment. Epileptic Disord 2006;8:285-8.

24. Saltik S, Uluduz D, Cokar O, Demirbilek V, Dervent A. A clinical and EEG study on idiopathic partial epilepsies with evolution into ESES spectrum disorders. Epilepsia 2005;46:524-33.

25. Pesantez-Rios G, Martinez-Bermejo A, Pesantez-Cuesta G. Neuropsychological alterations are frequent in rolandic epilepsy and its atypical developments. Rev Neurol 2016;63:125-9.

26. Berroya AG, McIntyre J, Webster $R$, et al. Speech and language deterioration in benign rolandic epilepsy. J Child Neurol 2004;19:53-8.

27. Gobbi G, Boni A, Filippini M. The spectrum of idiopathic Rolandic epilepsy syndromes and idiopathic occipital epilepsies: from the benign to the disabling. Epilepsia 2006;47 Suppl 2:62-6.

28. Northcott E, Connolly AM, Berroya A, et al. The neuropsychological and language profile of children with benign rolandic epilepsy. Epilepsia 2005;46:924-30.

29. van Klink NE, van 't Klooster MA, Leijten FS, et al. Ripples on rolandic spikes: a marker of epilepsy severity. Epilepsia 2016;57:1179-89.

30. Pal DK. Epilepsy and neurodevelopmental disorders of language. Curr Opin Neurol 2011;24:126-31.

31. Depienne C, Gourfinkel-An I, Baulac S, LeGuern E. Genes in infantile epileptic encephalopathies. In: Noebels JL, Avoli M, Rogawski MA, Olsen RW, Delgado-Escueta AV, eds. Jasper's Basic Mechanisms of the Epilepsies, 4th ed. Bethesda (MD) 2012.

32. Guerrini R, Parrini E. Neuronal migration disorders. Neurobio Dis 2010;38:154-66.

33. Reinthaler EM, Lal D, Jurkowski W, et al. Analysis of ELP4, SRPX2, and interacting genes in typical and atypical rolandic epilepsy. Epilepsia 2014;55:e89-93.

34. Royer B, Soares DC, Barlow PN, et al. Molecular evolution of the human SRPX2 gene that causes brain disorders of the Rolandic and Sylvian speech areas. BMC Genet 2007:8:72.

35. Lemke JR, Lal D, Reinthaler EM, et al. Mutations in GRIN2A cause idiopathic focal epilepsy with rolandic spikes. Nat Genet 2013:45:1067-72.

36. Reinthaler EM, Dejanovic B, Lal D, et al. Rare variants in gamma-aminobutyric acid type A receptor genes in rolandic epilepsy and related syndromes. Ann Neurol 2015;77:972-86.

37. Liu YT, Nian FS, Chou WJ, et al. PRRT2 mutations lead to neuronal dysfunction and neurodevelopmental defects. Oncotarget 2016;7:39184-96.

38. Djemie T, Weckhuysen $S$, Holmgren $P$, et al. PRRT2 mutations: exploring the phenotypical boundaries. I Neurol Neurosurg Psychiatry 2014;85:462-5.

39. Igarashi A, Okumura A, Shimojima $K$, et al. Focal seizures and epileptic spasms in a child with Down syndrome from a family with a PRRT2 mutation. Brain Dev 2016;38:597-600.

40. Nobile C, Striano P. PRRT2: a major cause of infantile epilepsy and oth- 
er paroxysmal disorders of childhood. Prog Brain Res 2014;213:141-58.

41. Lesca G, Rudolf G, Bruneau N, et al. GRIN2A mutations in acquired epileptic aphasia and related childhood focal epilepsies and encephalopathies with speech and language dysfunction. Nat Genet 2013:45:1061-6.

42. Dimassi $\mathrm{S}$, Labalme A, Lesca $\mathrm{G}$, et al. A subset of genomic alterations detected in rolandic epilepsies contains candidate or known epilepsy genes including GRIN2A and PRRT2. Epilepsia 2014;55:370-8.

43. Caraballo RH, Aldao Mdel R, Cachia P. Benign childhood seizure susceptibility syndrome: three case reports. Epileptic Disord 2011;13:133-9.

44. Cherian A, Baheti NN, Menon RN, et al. Atonic variant of benign childhood epilepsy with centrotemporal spikes (atonic-BECTS): a distinct electro-clinical syndrome. Brain Dev 2012;34:511-9.

45. Allen NM, Conroy J, Deonna T, et al. Atypical benign partial epilepsy of childhood with acquired neurocognitive, lexical semantic, and autistic spectrum disorder. Epilepsy Behav Case Rep 2016;6:42-8.

46. Hahn A, Pistohl J, Neubauer BA, Stephani U. Atypical "benign" partial epilepsy or pseudo-Lennox syndrome. Part I: symptomatology and long-term prognosis. Neuropediatrics 2001;32:1-8.

47. Doose H, Hahn A, Neubauer BA, Pistohl J, Stephani U. Atypical "benign" partial epilepsy of childhood or pseudo-lennox syndrome. Part II: family study. Neuropediatrics 2001;32:9-13.

48. Fejerman N, Caraballo R, Tenembaum SN. Atypical evolutions of benign localization-related epilepsies in children: are they predictable? Epilepsia 2000;41:380-90.

49. Kaga $M$, Inagaki $M$, Ohta R. Epidemiological study of Landau-Kleffner syndrome (LKS) in Japan. Brain Dev 2014;36:284-6.

50. Turner SJ, Morgan AT, Perez ER, Scheffer IE. New genes for focal epilepsies with speech and language disorders. Curr Neurosci Rep 2015;15:35.

51. Riccio CA, Vidrine SM, Cohen MJ, Acosta-Cotte D, Park Y. Neurocognitive and behavioral profiles of children with Landau-Kleffner syndrome. Appl Neuropsychol Child 2016:1-10.

52. Hughes JR. A review of the relationships between Landau-Kleffner syndrome, electrical status epilepticus during sleep, and continuous spike-waves during sleep. Epilepsy Behav 2011;20:247-53.

53. Paetau R. Magnetoencephalography in Landau-Kleffner syndrome. Epilepsia 2009;50 Suppl 7:51-4.

54. Duran MH, Guimaraes CA, Medeiros LL, Guerreiro MM. Landau-Kleffner syndrome: long-term follow-up. Brain Dev 2009;31:58-63.

55. Singhal NS, Sullivan JE. Continuous spike-wave during slow wave sleep and related conditions. ISRN Neurol 2014;2014:619079.

56. Caraballo RH, Veggiotti $P$, Kaltenmeier $M C$, et al. Encephalopathy with status epilepticus during sleep or continuous spikes and waves during slow sleep syndrome: a multicenter, long-term follow-up study of 117 patients. Epilepsy Res 2013;105:164-73.

57. Nickels K, Wirrell E. Electrical status epilepticus in sleep. Semin Pediatr Neurol 2008;15:50-60.

58. Raha S, Shah U, Udani V. Neurocognitive and neurobehavioral disabilities in Epilepsy with Electrical Status Epilepticus in slow sleep (ESES) and related syndromes. Epilepsy Behav 2012;25:381-5.

59. Schmitt B. Sleep and epilepsy syndromes. Neuropediatrics 2015;46:171-80.

60. Tuchman R. CSWS-related autistic regression versus autistic regression without CSWS. Epilepsia 2009;50 Suppl 7:18-20.

61. Filippini M, Boni A, Giannotta M, Gobbi G. Neuropsychological development in children belonging to BECTS spectrum: long-term effect of epileptiform activity. Epilepsy Behav 2013;28:504-11.

62. Liukkonen $E$, Kantola-Sorsa $E$, Paetau $R$, et al. Long-term outcome of 32 children with encephalopathy with status epilepticus during sleep, or ESES syndrome. Epilepsia 2010;51:2023-32.

63. van den Munckhof B, van Dee V, Sagi L, et al. Treatment of electrical status epilepticus in sleep: a pooled analysis of 575 cases. Epilepsia 2015:56:1738-46.

64. McTague A, Cross JH. Treatment of epileptic encephalopathies. CNS Drugs 2013;27:175-84.

65. Veggiotti $P$, Pera MC, Teutonico F, et al. Therapy of encephalopathy with status epilepticus during sleep (ESES/CSWS syndrome): an update. Epileptic Disord 2012;14:1-11. 